Tohoku J. exp. Med., 1967, 92, 389-401

\title{
Studies on Light Reflection of Blood in the Application of Fiber Optics*
}

\author{
Kinji Ishikawa \\ The First Department of Internal Medicine (Prof. T. Nakamura), \\ Tohoku University School of Medicine, Sendai
}

\begin{abstract}
The mechanism of light reflection in blood, which is the underlying principle for the use of fiber opties in in vivo densitometry or oximetry, was investigated theoretically and experimentally using bovine blood. The results are summarized as follows. 1) Theoretically, the intensity of light reflected, $I$, was represented by a formula: $I=I_{0} \times e^{-2 K C} \times[(1-\rho), \rho] \times r \times a$, where $I_{0}, K, C, \rho$, and $\alpha$ were incident light, extinction coefficient of plasma, hematocrit and reflexibility of blood, respectively; $r$ is the length of the side of a cube when the red blood cell was assumed as of cubic form. 2) Light reflection was proved to take place only in the region several microns apart from the tip of fiber optics. 3) Reflected light was increased with increase in hematocrit up to $60 \%$ but was decreased with further increase in hematocrit. This phenomenon was interpreted as being due to so-called 'shading effect' resulting from rescattering of reflected light by red blood cells. 4) $\alpha$ was calculated as $3.4 \%$. Low reflexibility played a major part in decrement of incident light.
\end{abstract}

The indicator dilution technique has been used as a valuable tool for diagnosis of cardiovascular diseases. Recently, attention has been directed to engaged in the application of fiber optics ${ }^{1}$ to in vivo oximetry or densitometry. ${ }^{2-5}$ Oxygen saturation or dye concentration can be estimated immediately without blood sampling with the aid of fiber optics. However, information is still incomplete concerning the theoretical treatment and fundamental phenomena of light reflection from blood.

There have been several reports studying light reflection in which light was introduced into blood from the outside of blood and light reflection from air blood boundary was investigated. ${ }^{6-10}$ These observations are invalid, for the estimation of light reflection in the fiber optics in which light is introduced directly into the inside of blood.

It is essential to settle the fundamental problems involved in light reflection in blood before fiber optics are applied to clinical investigations. The purpose of the present investigation is to make theoretical approach and to investigate the fundamental phenomena regarding light reflection in blood.

Received for publication, March 22, 1967.

* Part I, Theoretical Considerations.

This investigation was supported in part by U.S. Public Health Service Research Grant HE 08018-04 from National Heart Institute. 


\section{Theoretical Considerations}

When light passes from a certain medium to another, light reflection will occur according to Frennel's law. If the refractive indexes of the two media are $n_{1}$ and $n_{2}$, reflexibility is given by $\left(\begin{array}{c}n_{1}-n_{2} \\ n_{1}+n_{2}\end{array}\right)^{2}$. Accordingly, light reflection by fiber optics will take place from the boundaries of three different media; fiber optics, plasma and blood corpuscles. It is evident that reflection occurs from red blood cells, since light reflection is not observed after hemolysis or when blood corpuscles are removed.

\section{Ideal case}

The problem of measurement in situ is most conveniently approached by means of a gross simplification. Incident light from an afferent fiber passes through plasma and reaches red blood cells located at different distances. A part of light which reaches the cells is reflected and returns to an efferent fiber. When a parallel monochromatic light passes throngh plasma, the intensity of light is decreased according to Lambert-Beer's law. The distance from fiber optics to red blood cells is different according to different individual cells. The mean of the distances is defined as 'mean reflection distance', $d$, and the following equations can be derived as a first approximation (Fig. 1):

$$
\begin{aligned}
& I_{1}=I_{0} \times e^{-K C d} \\
& I_{2} / I_{1}=a \\
& I=I_{2} \times e^{-K C d},
\end{aligned}
$$

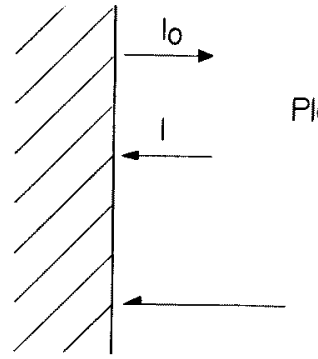

Afferent and efferent fibers

$I_{0}$ : Incident light

$I$ : Reflected light

$d$ : Mean reflection distance

a: Reflexibility

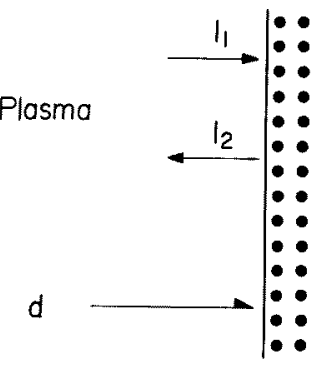

Red blood cell

$I_{1}=I_{0} \times e^{-K C d}$

$I_{2} / I_{1}=\alpha$

$I=I_{2} \times e^{-K C d}$

$I=I_{0} \times e^{-2 K C d} \times a$

Fig. 1. Illustration for the mathematical expression giving the intensity of reflected light. The distances from fiber optics to red blood cells are different according to individual corpuscles, and the concept of mean of distances is introduced (mean reflection distance; d). Lambert-Beer's law holds on the decrease in light through plasma. When the reflexibility of red blood cell is given, the equation (1) can be derived. 
where $I_{0}, I_{1}, I_{2}$, and $I$ are intensities of incident light, of light which reaches red blood cells, of light which is reflected by the cells, and of reflected light which gets to efferent fibers, respectively. The ratio of light reflection by the cell is defined as 'reflexbility', $\alpha . \quad K$ represents extinction coefficient of plasma, and $C$ the concentration of plasma which is regarded as 1 in usual cases. Combining these three equations, the following formula can be obtained:

$$
I=I_{0} \times e^{-2 K C d} \times \alpha
$$

\section{Calculation of mean reflection distance}

Assumption is made that a red blood cells is a cube with edges of $r$ in length. Suppose now a columnar space made by serially arranged cubes of the abovementioned dimension as shown in Fig. 2. A red cell may occupy any one of these

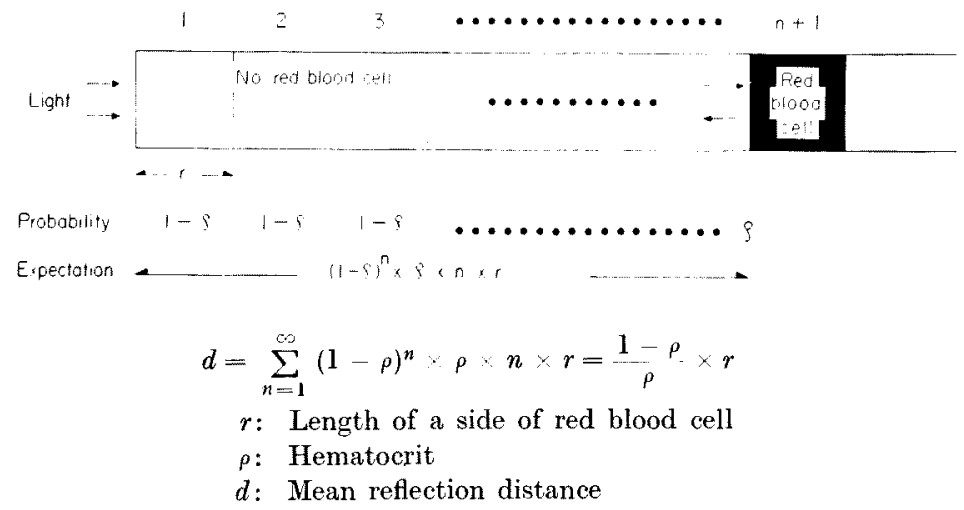

Fig. 2. Calculation of mean reflection distance. The red blood cell is assumed to be a regular hexahedron, of which edges are $r$ in length. If the probability that one of the serially arranged cubes with edges of $r$ in length is occupied by the red blood cell is $\rho$, the probability that the cube is unoccupied is $1-p$. The probability that a ray passes the cubes from No. 1 to No. $n$, is $(1-p)^{n}$, and the expectation that the ray is reflected at the cube No. $n+1$ is $(1-\rho)^{n} \times \rho \times n \times r$. The mean reflection distance is calculated as $\frac{1-\rho}{\rho} \times r$.

cubes. The probability that incident light passes through unoccupied cubes from No. 1 to No. $n$ is $(1-\rho)^{n}$, and the expectation that the light is reflected by a red cell occupying the cube No. $n+1$ is given by $(1-\rho)^{n} \times \rho \times r \times n$. Accordingly, the mean reflection distance can be calculated as follows:

$$
d=\sum_{n=1}^{\infty}(1-\rho)^{n} \times \rho \times n \times r=\frac{1-\rho}{\rho} \times r .
$$

Fig. 3 indicates the relationship between mean reflection distance calculated by this equation and hematocrit. Combining equations (1) and (2), we obtain:

$$
I=I_{0} \times e^{2 K C \times[(1-\rho) / n] \times r} \times a
$$

The relationship between the intensity of reflected light calculated by this equa- 


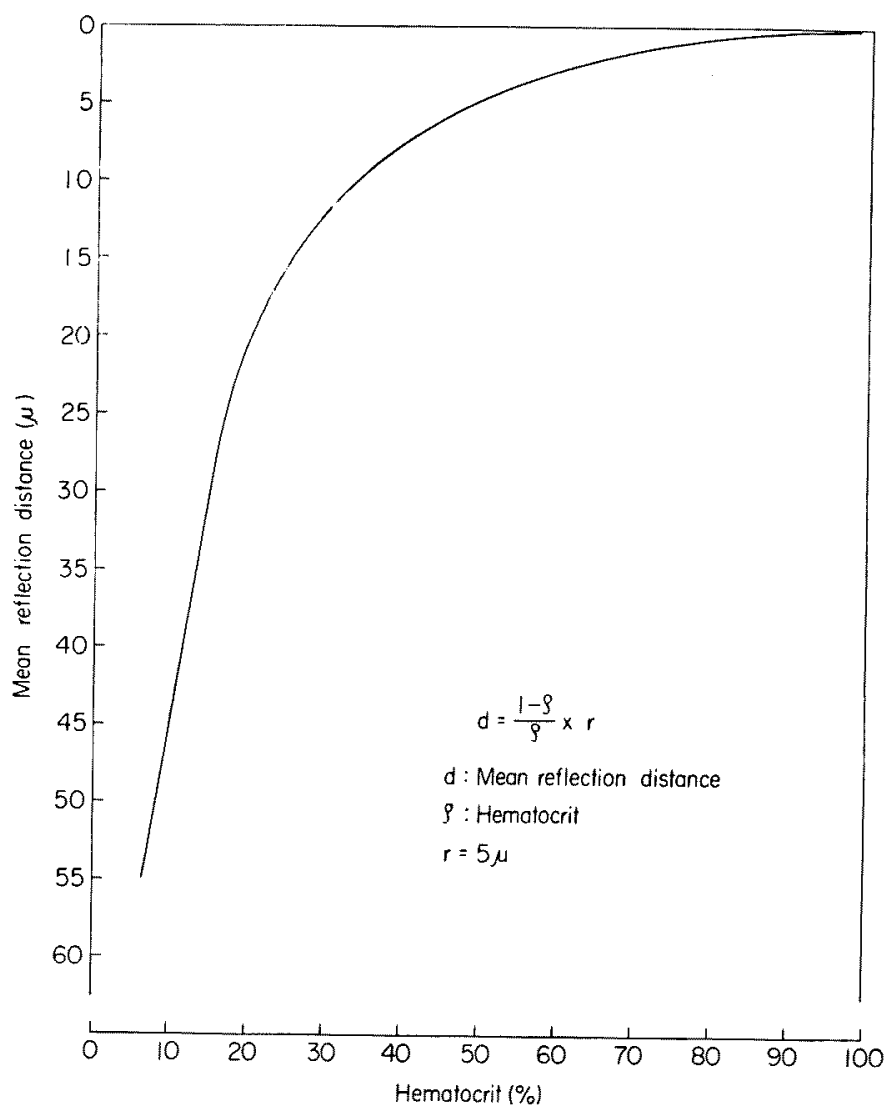

Fig. 3. Relationship between mean reflection distance and hematocrit. Mean reflection distance at hematocrit 50 per cent is 5 microns. Note how light is reflected at the close vicinity of the fiber optics.

tion and hematocrit is shown in Fig. 4.

Calculation of reflexibility

From equation (3), reflexibility can be simply obtained when hematocrit is 100 per cent or plasma concentration is zero, and then,

$$
\alpha=\frac{I}{I_{0}}
$$

Another more convenient form is given by employing equation (1) as:

$$
\log \frac{I_{-}}{I_{0}^{-}}=-2 K C d+\log \alpha .
$$

This represents a linear regression on semilog paper between reflected light and plasma concentration and the intercept of the ordinate gives reflexibility. 


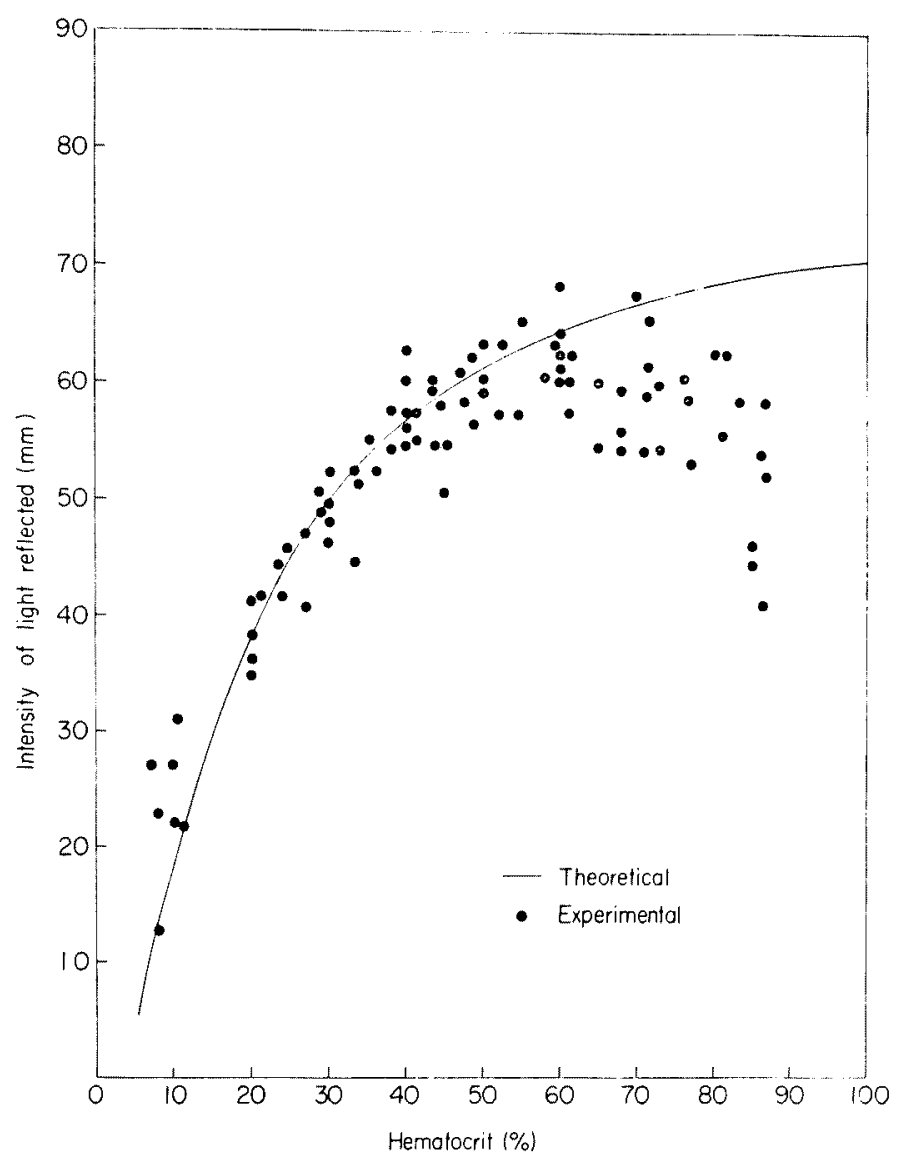

Fig. 4. Relationship between hematocrit and reflected light.

\section{Materials and Methods}

Bovine blood was used in the following experiments. Blood was heparinized at a concentration of 1,000 units per liter, and was equilibrated with 100 per cent oxygen to maintain oxygen saturation over 95 per cent. The blood was not defibrinated. Hematocrit of blood was adjusted as follows. Red blood cells obtained by centrifugation at $3,000 \mathrm{cpm}$ for 30 minutes were remixed with supernatant plasma so as to give a required hematocrit. Dilution of plasma was made for measurement of reflexibility. A certain amount of supernatant plasma by centrifugation was replaced by the same amount of physiologic saline, so that the plasma was diluted to a desired concentration. All the experiments were performed at room temperature, 12 to 48 hours after blood sampling. Hemolyzed blood was discarded.

Blood sample was drawn through a metal tube at a constant speed of $5.6 \mathrm{~cm} /$ sec $(0.9 \mathrm{ml} / \mathrm{sec})$ with a constant aspirator, and fiber optics (Nippon Glass Fiber Co., 


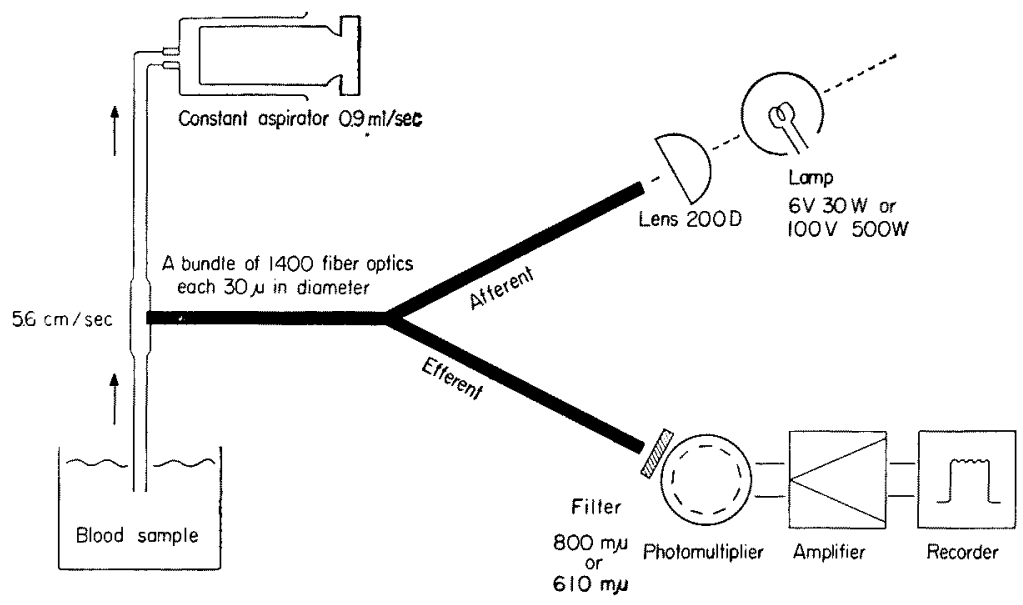

Fig. 5. Diagrammatic representation of the fiber optics recorder system.

Ltd., Tsu, Mie, Japan), each fiber of which was 30 microns in diameter and 100 centimeters in length, were used. The number of aperture was 0.55. One end of fiber optics was divided into two channels of afferent and efferent bundles. The fibers in two channels were randomly mixed at the other end to ensure a good sampling of reflected light. A glass rod, 8 millimeters in diameter, 50 centimeters in length and covered with aluminium leaf, was used for a special purpose of investigating the 'shading effect'. One end of the glass rod was inserted into blood and the other end was divided into afferent and efferent limbs.

Light from a tungsten lamp, 6 volts 30 watts or 100 volts 500 watts, was focused with a lens of 200 dioptries on the proximal end of the afferent bundle. The reflected light that passes through a filter was detected by a photomultiplier tube, and output signal was fed into Sanborn Twin Viso Cardiette (Sanborn Co., Ltd., Cambridge, Mass., U.S.A).

A photomultiplier R 196 (Hamamatsu Television Co., Ltd., Hamamatsu, Shizuoka, Japan) which had the maximum spectral response of $800 \mathrm{~m} \mu$ with a range of 400 to $1,200 \mathrm{~m} \mu$ was used. A filter which had the maximum transmission at $800 \mathrm{~m} \mu$ with a half-intensity band width of $100 \mathrm{~m} \mu$ was used.

The intensity of light reflected was expressed in terms of deflection of the recorder $(\mathrm{mm})$ at an arbitrary attenuation. The 90 per cent response time of the fiber opties recorder system was 0.25 second.

\section{Results}

\section{1) Relationship between intensity of light reflected and hematocrit}

Ninety bovine blood samples obtained from 7 animals were used. Hematocrit was varied from 7 to 87 per cent. The results are shown in Fig. 5. The intensity of reflected light was increased with the increase of hematocrit and reached its maximum when hematocrit was 60 per cent. Further increase in hematocrit 


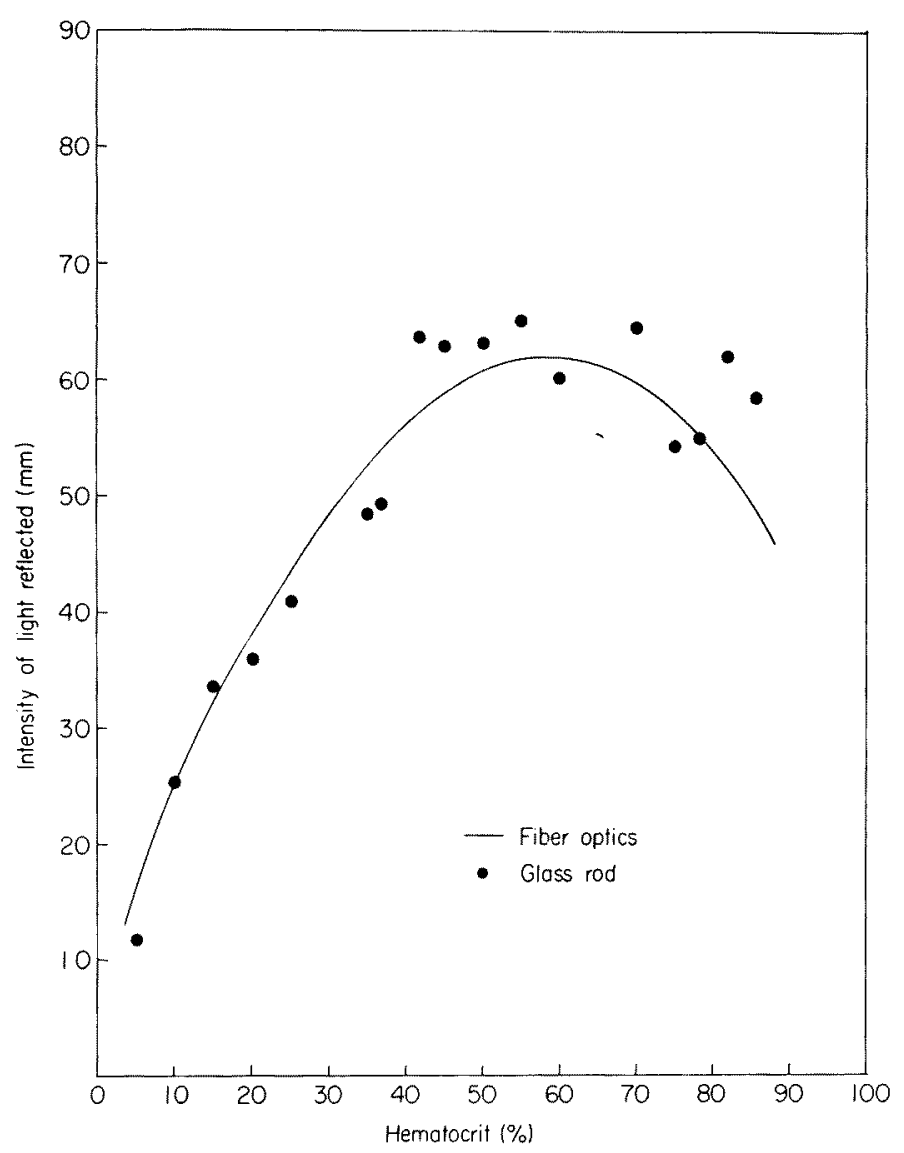

Fig. 6. Comparison of reflected light with the fiber optics and the glass rod.

decreased reflected light.

Another experiment was performed with a glass rod to investigate the shading effect. The relationship between hematocrit and reflected light with the glass rod did not show any significant difference as compared with the relationship with fiber optics as shown in Fig. 6.

\section{2) Measurement of reflexibility}

Fourteen bovine blood samples were prepared from 10 animals. The values of plasma concentration and reflected light are given in Table 1. When logarithm of reflected light was plotted against plasma concentration, a linear regression was demonstrated, and the validity of equation (5) was confirmed. Thus the intercept of the ordinate was easily obtained by extrapolation of the regression line. One of the examples is shown in Fig. 7. Light reflexion was assessed by inserting the fiber optics into mercury and the intensity of reflexion was regarded as equal to that of incident light, based on the assumption that reflexibility of mercury was 100 per 
TABLE 1. Plasma concentration and intensity of light reflected

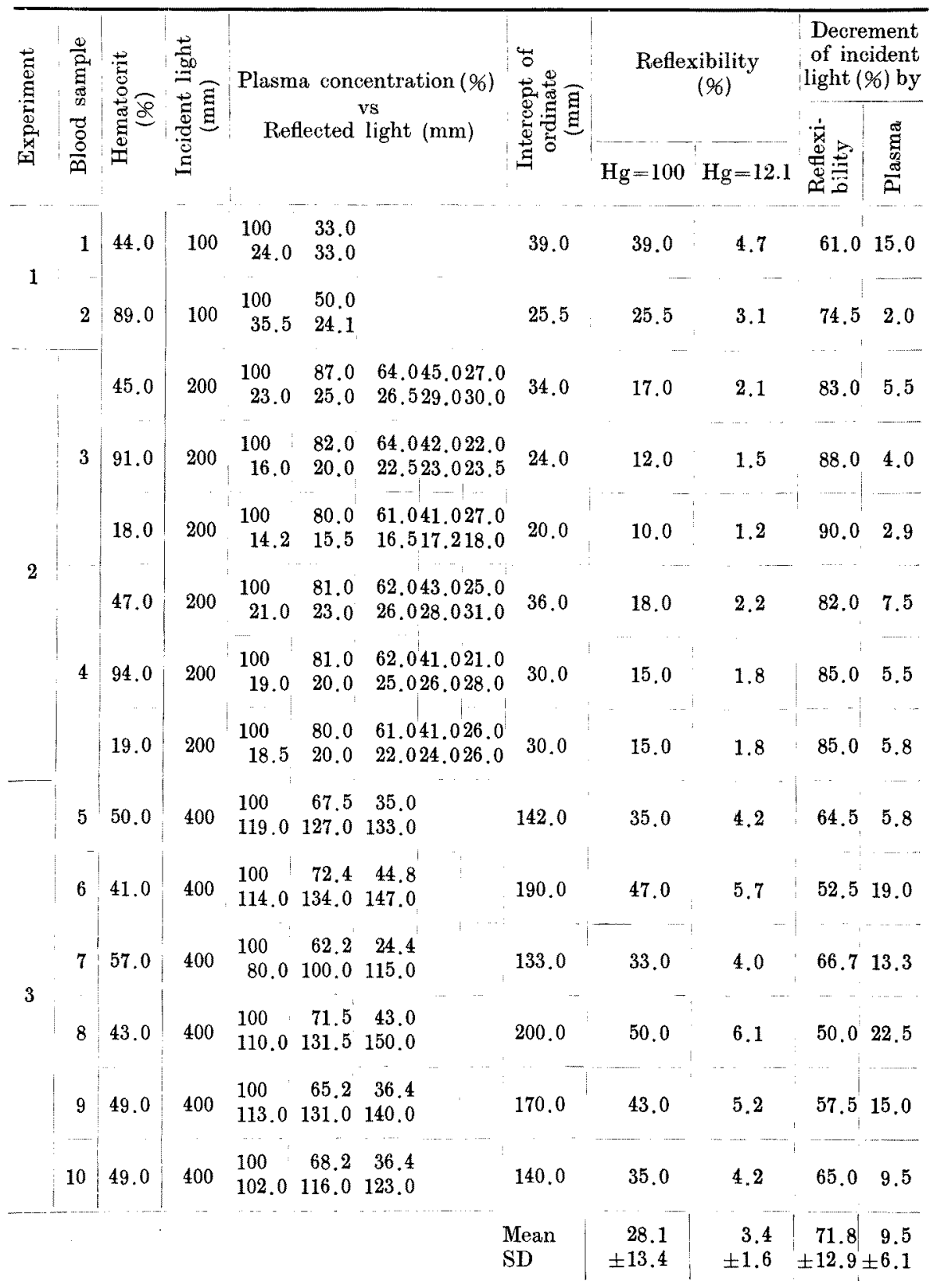

cent. Reflexibility of blood was calculated using equation (4), and it was $28.1 \pm 13.4$ per cent of that of mercury. As the reflexibility of mercury was in reality 12.1 per cent, that of blood was $3.4 \pm 1.6$ per cent. Decrement of incident light was divided into one by passage through plasma and another by reflexibility. The former was 


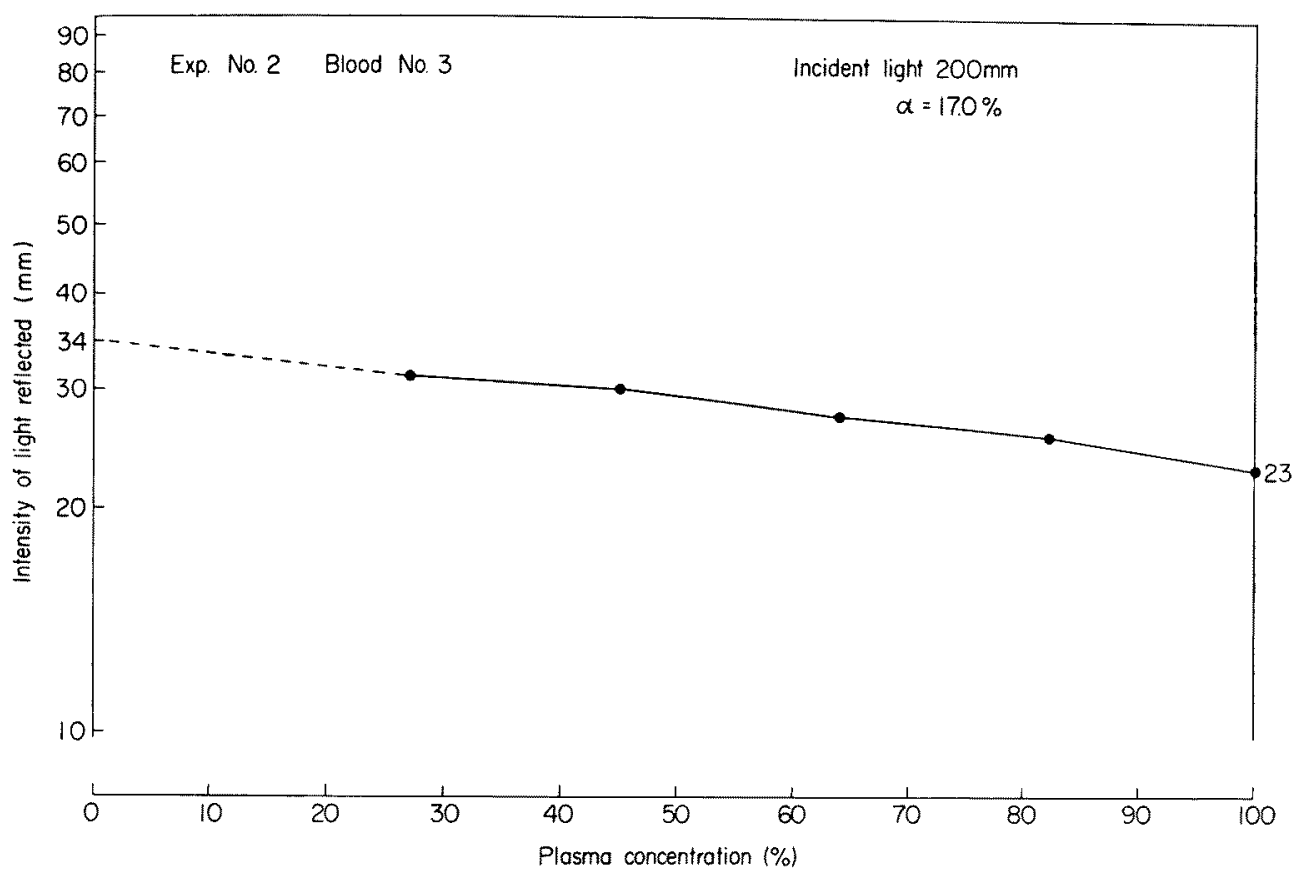

Fig. 7. Graphic representation of plasma concentration and reflected light. Logarithm of reflected light is plotted against plasma concentration. A linear regression is observed. The intercept of the ordinate is obtained by extrapolation of the regression line. The intercept of the ordinate $(=34)$ represents reflected light when plasma concentration is zero. Reflected light from mercury is 200 . Employing equation (4), the reflexibility is calculated as $\begin{gathered}34 \\ 200\end{gathered} \times 100=17.0 \%$. Decrement in reflected light by reflexibility is calculated as $200-34 \times 100=83.0 \%$. Decrement by plasma is calculated as $\begin{gathered}34-23 \\ 200\end{gathered} \times 100=5.5 \%$.

calculated as $9.5 \pm 6.1$ per cent and the latter was $71.8 \pm 12.9$ per cent. It appeared from these considerations that a decrement of incident light occurred mainly when light was reflected by red blood cells.

\section{Discussion}

\section{1) Relationship between the intensity of reflected light and hematocrit}

Reflected light may increase with the increase of hematocrit so long as light reflection takes place at red blood cells. The equation (3) demonstrates that reflected light increases as hematocrit increases. Comparison was made between theoretical and experimental values in Fig. 4. Theoretical values were in good agreement with experimental values when hematocrit was lower than 60 per cent. But, reflected light determined experimentally decreased, when hematocrit further increases. This result was the same as in the report by Enson et al. ${ }^{11}$ This 

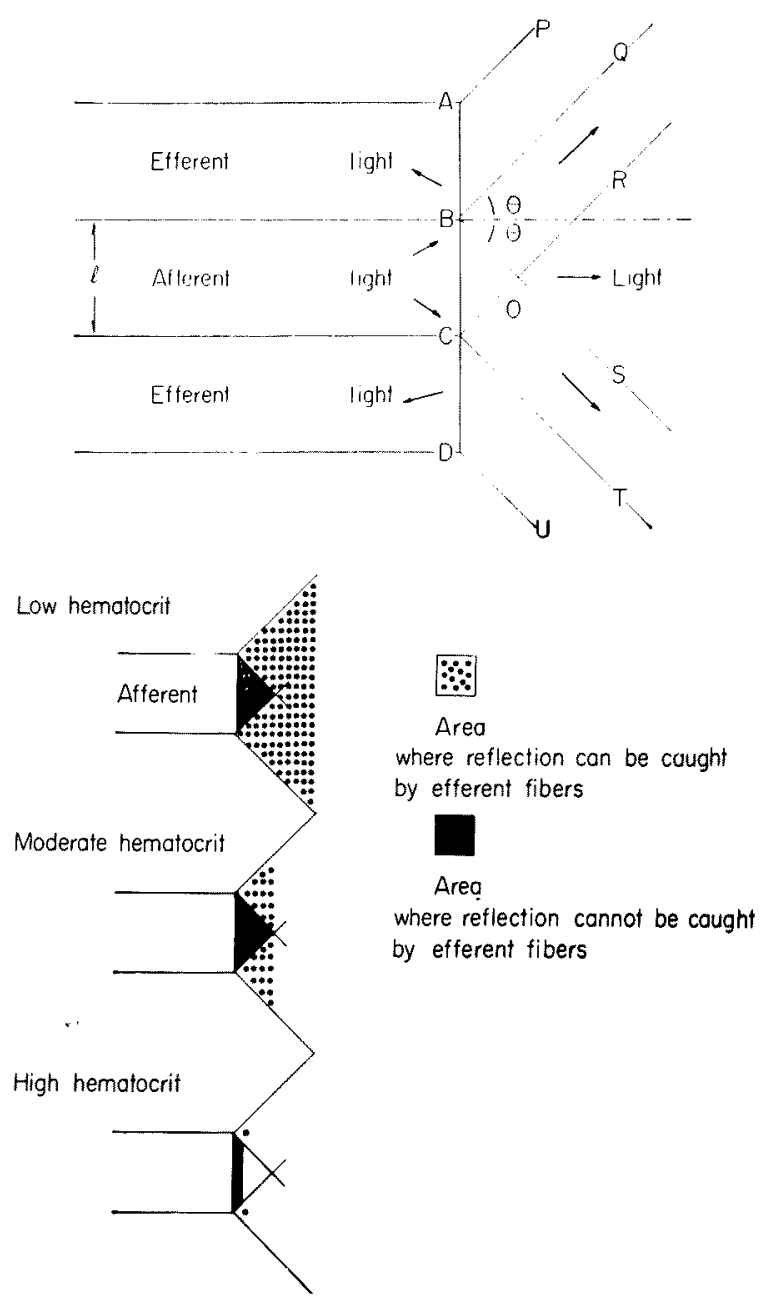

Fig. 8. Schematic presentation of shading effect A. Reflection occurring adjacent to an afferent fiber (BOC) cannot be caught by efferent fibers. This region (blackened area) is proportionally small in low hematocrit but becomes large with high hematocrit.

discrepancy was explained as follows.

As the fiber optics used for this experiment were composed of afferent and efferent fibers, afferent fibers cannot catch reflected light. The fiber optics have a certain region where incident light through the fibers can emerge and where reflected light can be caught. This region can be expressed in terms of angle $\theta$ which is determined by the number of aperture of fiber optics as shown in Fig. 8 . Afferent fibers can illuminate the area QBCT in Fig. 8. The near-by efferent fibers can catch reflected light from the region $\mathrm{RCDU}$ or PABS, while reflected light adjacent to the afferent fiber $\mathrm{BCO}$ cannot be caught by any efferent fibers 
('shading effect A'). In cases of low hematocrit, reflection takes place from a wide region, since mean reflection distance is lengthened. The area where reflection cannot be caught amounts to only a small portion and the shading effect $\mathrm{A}$ is negligible. When hematocrit is high, however, reflection takes place adjacent to the afferent fiber and almost all reflection occurs in the shaded area. Accordingly, reflected light caught by the efferent fibers will decrease as hematocrit increases over a certain value. The shaded area is determined by the angle $\theta$ and the diameter of afferent fibers $l$.

Shading effect A will play a great role in decreasing reflected light, if the angle $\theta$ is small and the diameter $l$ is large. The fiber optics used in this study have a diameter of 30 microns and an angle of nearly 45 degrees.

Experiments with a glass rod where afferent and efferent pathways at one end are the same were performed to investigate the shading effect A. As shown in Fig. 6, there was no significant difference between the values obtained with fiber optics and glass rod. It was concluded that shading effect $A$ had a little effect if the diameter remains smaller than 30 microns and the angle $\theta$ larger than 45 degrees.

There have been many reports in regard to the turbidity or scattering effects of the colloidal system. It is evident that light is scattered by colloid particles, and the scattering per unit volume is proportional to the number of colloid particles. But it is a well known phenomenon that scattering of light decreases if the concentration of colloid particles exceeds a certain limit. ${ }^{12-14}$ It has been explained that scattered light at a high concentration will be scattered again before it reaches the detector and escapes estimation. ${ }^{15,16}$ This is quite similar to the phenomenon now in problem.

One of the basic assumptions required in deriving the working equation is that all reflected light can reach the detector, but a part of reflected light will be shaded off by red blood cells on the way back to the detector ('shading effect B'). The possibility that reflected light will be caught by red blood cells will increase as hematocrit increases. The probability that reflected light can get to the efferent fiber when reflected light traverses the distance of $n \times r$ is shown as $(1-\rho)^{n}$. Accordingly, reflected light will decrease when hematocrit increases further over a certain value. It was considered that the shading effect $B$ will play a great role in reducing reflected light when hematocrit is over than 60 per cent.

\section{2) Measurement of reflexibility}

There was a linear relationship between plasma concentration and logarithm of reflected light and the validity of equation (5) was confirmed. The reflexibility between plasma and red blood cell was 28.1 per cent of that of mercury, and it was 3.4 per cent after correction with the reflexibility of mercury was made. It was demonstrated that only a small portion of incident light was reflected. This is due to small difference in refractive indexes of plasma and red blood cell. There is a report that blood became transparent as a whole when plasma protein was increased and refractive index was raised up to that of the blood cell. ${ }^{17}$ We must realize the technical difficulties originating from this fact in recording dye-dilution curve hy 
fiber optics, that is, the reflexibility is so low and signal-to-noise ratio will be easily decreased by a little change in reflexibility. The marked decrement of incident light by blood is mainly due to this low reflexibility, and decrement by plasma is considerably small. Ten per cent changes in reflexibility is far more greater influence than the change in reflected light produced with $10 \mathrm{mg} / \mathrm{l}$ of Cardio green. This is the reason why excessive flucutation or pulsation of base line is inevitable in recording a dye dilution curve.

\section{Summary}

1) A theoretical approach was attempted to define the intensity of light reflected in blood when fiber optics were used, and the following mathematical expression was derived:

$$
I=I_{0} \times e^{2 K C \times[(1-\rho) / \rho] \times r} \times \alpha .
$$

2) This formula was valid over a certain range of hematocrit. Reflected light decreased, however, when hematocrit exceeded 60 per cent. This was explained by the shading effects.

3) The reflexibility of light between plasma and red blood cell amounted to 3.4 per cent. A major portion of decrement of incident light resulted from this low reflexibility, and small variation in reflexibility produced a great variation in reflected light.

\section{Acknowledgment}

I wish to thank Dr. T. Nakamura, Professor of Medicine, Tohoku University School of Medicine, for his direction and I am grateful for the invaluable cooperation of Drs. R. Katori, K. Miyazawa, Y. Sendo, M. Yamaki, and Y. Kobayashi, the Ist Department of Internal Medicine, Tohoku University School of Medicine, and for the technical assistance of Miss K. Handa. Also I am indebted to Dr. C. Watari, Assistant Professor of Mathe. matics, and Dr. M. Hirai, Assistant Professor of Physics, Tohoku University, for their valuable suggestions.

\section{References}

1) Kapany, N.S. Fiber optics. Part I. Optical properties of certain dielectric cylinders. J. Opt. Soc. Amer., 1957, 47, 413-422.

2) Enson, Y., Jameson, A.G. \& Cournand, A. Intracardiac oximetry in congenital heart disease. Circulation, 1964, 29, 499-507.

3) Gamble, W.J., Hugenholtz, P.G., Monroe, R.G., Polanyi, M. \& Nadas, A.S. The use of fibsr optics in clinical cardaic catheterization. I. Intracardiac oximetry. Circulation, 1965, 31, 328-343.

4) Hugenholtz, P.G., Gamble, W.J., Monroe, R.G. \& Polanyi, M. The use of fiber optics in clinical cardiac catheterization. II. In vivo dye-dilution curves. Circulation, 1965, 31, 344-355.

5) Gamble, W.J. \& Innis, R. Visualization of intracardiac structures by eatheterization with a flexible fiber optic instrument. Fed. Proc., 1965, 24 (£2 part 1), 156.

6) Amy, L. Sur la couleur des corps par réflexion. I. Etude théorique. Revue d'Optique, $1937,16,81-85$. 
7) Sannié., C., Amy, L. \& Sarraf, J.-M. Sur la conleur des corps par réflexion. II. Vérifications expérimentales. Revue d'Optique, 1937, 16, 86-92.

8) Dognon, A., Loeper, J. \& Housset, E. Etude optique de l'agrégation réversible des hématies. C.r. Soc. Biol. (Paris), 1949, 143, 769-771.

9) Rodrigo, F.A. The determination of the oxygenation of blood in vitro by using reflected light. Amer. Heart J., 1953, 45, 809 822 .

10) Dognon, A.\& Suquet, P. Sur le pouvoir réflecteur du sang. C.r. Soc. Biol. (Paris), 1957, $151,1111-1113$.

11) Enson, Y., Briscoe, W.A., Polanyi, M.L. \& Cournand, A. In vivo studies with an intravascular and intracardiac reflection oximeter. J. appl. Physiol., 1962, 17, 552558 .

12) Teorell, T. Photometrische Messung der Konzentration und Dispersität in kolloiden Lösungen. III. Kolloid Z., 1931, 54, 150-156.

13) Katsurai, T. Uber das Tyndallicht von Milch, Eisenchlorid und AluminiumchloridLösungen. Kolloid Z., 1934, 67, 143-145.

14) Katsurai, T. Notiz zu dem Tyndallicht der Milch. Kolloid Z., 1936, 74, 287-288.

15) Caspersson, T. Zur Kenntnis der Optik weisser Sole. II. Die diffuse Seitenstrahlung. Kolloid Z., 1933, 65, 162-170.

16) Duysens, L.N.M. The flattening of the absorption spectrum of suspensions, as compared to that of solutions. Bioch. Biophys. Acta., 1956, 19, 1-12.

17) Barer, R. Spectrophotometry of clarified cell suspensions. Science, 1955, 121, 709715 . 